\title{
A novel feature extraction method of eddy current testing for defect detection based on machine learning
}

DOI:

10.1016/j.ndteint.2019.04.005

\section{Document Version}

Accepted author manuscript

Link to publication record in Manchester Research Explorer

\section{Citation for published version (APA):}

Tao, Y., Yin, W., Xu, H., \& Salas Avila, J. R. (2019). A novel feature extraction method of eddy current testing for defect detection based on machine learning. NDT \& E International. https://doi.org/10.1016/j.ndteint.2019.04.005

\section{Published in:}

NDT \& E International

\section{Citing this paper}

Please note that where the full-text provided on Manchester Research Explorer is the Author Accepted Manuscript or Proof version this may differ from the final Published version. If citing, it is advised that you check and use the publisher's definitive version.

\section{General rights}

Copyright and moral rights for the publications made accessible in the Research Explorer are retained by the authors and/or other copyright owners and it is a condition of accessing publications that users recognise and abide by the legal requirements associated with these rights.

\section{Takedown policy}

If you believe that this document breaches copyright please refer to the University of Manchester's Takedown Procedures [http://man.ac.uk/04Y6Bo] or contact uml.scholarlycommunications@manchester.ac.uk providing relevant details, so we can investigate your claim.

\section{OPEN ACCESS}




\section{Accepted Manuscript}

A novel feature extraction method of eddy current testing for defect detection based on machine learning

Liyuan Yin, Bo Ye, Zhaolin Zhang, Yang Tao, Hanyang Xu, Jorge R. Salas Avila, Wuliang Yin

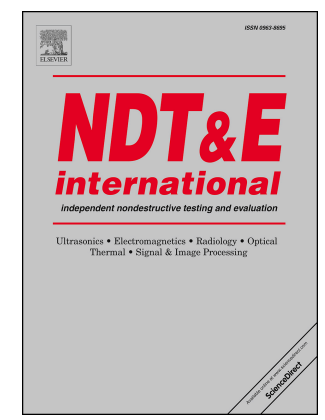

PII: S0963-8695(18)30612-1

DOI: https://doi.org/10.1016/j.ndteint.2019.04.005

Reference: $\quad$ JNDT 2108

To appear in: $\quad N D T$ and $E$ International

Received Date: 19 October 2018

Revised Date: 24 March 2019

Accepted Date: 8 April 2019

Please cite this article as: Yin L, Ye B, Zhang Z, Tao Y, Xu H, Salas Avila JR, Yin W, A novel feature extraction method of eddy current testing for defect detection based on machine learning, NDT and $E$ International (2019), doi: https://doi.org/10.1016/j.ndteint.2019.04.005.

This is a PDF file of an unedited manuscript that has been accepted for publication. As a service to our customers we are providing this early version of the manuscript. The manuscript will undergo copyediting, typesetting, and review of the resulting proof before it is published in its final form. Please note that during the production process errors may be discovered which could affect the content, and all legal disclaimers that apply to the journal pertain. 


\title{
A Novel Feature Extraction Method of Eddy Current Testing for Defect Detection Based On Machine Learning
}

\author{
Liyuan Yin ${ }^{\mathrm{a}}$, Bo Ye ${ }^{\mathrm{a}}$, Zhaolin Zhang ${ }^{\mathrm{a}}$, Yang Tao ${ }^{\mathrm{b}, *}$, Hanyang $\mathrm{Xu}^{\mathrm{b}}$, \\ Jorge R. Salas Avila ${ }^{\mathrm{b}}$, Wuliang Yin ${ }^{\mathrm{b}}$ \\ ${ }^{a}$ Faculty of Information Engineering and Automation, Kunming University of Science and \\ Technology, Kunming, China, 650500 \\ ${ }^{b}$ School of Electrical and Electronic Engineering, University of Manchester, Manchester, \\ United Kingdom, M13 9PL
}

\begin{abstract}
In eddy current testing, the trajectory of the impedance data due to a defect is presented as a Lissajous curve (LC) in the complex plane. This paper proposes a novel analytical model for describing a LC. Further, a new feature extraction method is implemented which automatically computes four geometric features (amplitude, width, angle and symmetry) from Lissajous figures. In addition, six machine learning-based classifiers are used for automatic defect identification based on these features. High detection rates are achieved for both the simulated and experimental data, which demonstrates the flexibility of the analytical model and the validity of the methodology.
\end{abstract}

Keywords: Eddy current testing, Lissajous figure, Feature extraction, Machine learning

\section{Introduction}

Eddy current testing (ECT) is a low-cost non-destructive testing method that has been widely applied to the detection of crack and defect in conductive materials $[20,25]$. Recent studies on automatically detecting defects by ECT tend to rely on machine learning (ML) algorithms $[32,23,22,3,1]$. Many of these ML-motivated studies entail a feature space as the input for the training of ML models. The features can be extracted from the impedance signal obtained by ECT sensors using various analytical methods.

Time-domain feature extraction methods analyse the impedance signal directly. Commonly harnessed time-domain features in pulsed ECT (PECT) include the rising time, rise-to-peak time, slope, and peak amplitude, etc. [30] which in general correlate to the size, depth and other properties of defect [6]. Moreover, principle component analysis (PCA) and its variations are extensively

\footnotetext{
* Corresponding author

Email address: yang.tao@manchester.ac.uk (Yang Tao)
} 
exploited to extract features from the time-domain signal [4]. For example, the research in [11] employed PCA to the differential time response signal resulting in defect-related features based on which surface and subsurface defects under different interlayer gaps and lift-off effects were detected.

In terms of frequency-domain features, they are often obtained by conducting a fast Fourier transform (FFT) to the response signal. In [10], PECT imaging and frequency spectrum analysis were successfully applied to detect, classify, and evaluate the sub-surface defects under the influence of edge effect of specimen. Furthermore, time-frequency analysis such as wavelet transform $[29,17]$ and Rihaczek distribution [12], etc. have shown their effectiveness in recovering defect-related features from time-domain signal and spectral response. Additionally, the feature space can be generated by fitting the response signal with non-linear approximation functions. In [5], summed Gauss function was utilised to approximate the spectrum of response signal, and features were extracted by fitting the function to different defect responses.

In addition to the time and frequency domain methods, image recognition is also an effective technique for feature extraction. In ECT, the trajectory of impedance data due to a defect is presented as a Lissajous curve (LC) in the complex plane. The shape of the LC is determined by the sensor geometry, relative motion between the sensor probe and specimen, the electromagnetic properties of the specimen, the profile of defect, and some other possible factors. The excitation frequency is a key factor of the LC shape. As a consequence, the distinguishing profile of defect for the same ECT sensor system would be embedded as the geometric features in the resultant LC [7]. These geometric features can be extracted using image recognition algorithms and then be used as the inputs of ML algorithms for further classification tasks. In [8], Content-Based Image Retrieval (CBIR) technique was adopted to extract geometric features from LCs for defects of aircraft specimen. Several features such as the perimeter, area, centre of gravity, inclination angle, inertia principal axes, wideness, length, and symmetry of the LC are generated using CBIR. The same method was latter harnessed in another study [9], in which the geometric features were extracted for a few measurements considering different orientations between the sensor probe path and the defect. The advances of modern hardware capability enable training a machine-learning model with data capturing various types of defects. In the circumstance of limited resources, however, acquiring such a set of real experimental data might be expensive which gives rise to the research of data augmentation techniques.

In this paper, an analytical model is proposed for the description of LCs, and by tuning the parameters of the model, an artificial dataset could be generated for training ML models. As long as the LC resulted from practical measurement of defect belongs to the distribution of the simulated artificial feature space, the trained ML models would be able to make a discrimination of the defect. Moreover, a clustering-based algorithm is proposed to extract the geometric features of LC which is simple and easy to implement. Lastly, the simulated feature space is fed to six ML algorithms, and the trained models are tested with data of practical measurements of defect. High detection rates 
demonstrate the validity of the proposed analytical model and the clusteringbased feature extraction method. The remainder of the paper is organised as follows. Section 2 introduces the analytical equations used to describe the LCs on the complex impedance plane and shows how the parameters of the equations influence the geometrical features of the LC. The clustering-based feature extraction algorithm is presented in Section 3. Section 4 describes the experimental system including a customised sensor, 2-D motorised scanning stage, field-programable gate array (FPGA) and host PC. Section 5 demonstrates the detailed procedures of conducting machine learning algorithms, and the results are presented and discussed in Section 6. The last section concludes the paper.

\section{Analytical Model for Lissajous Curve}

When an ECT probe crosses a defect in the test specimen, the resultant impedance signal in the complex plane has a shape similar to the digit ' 8 '. One 'petal' is developed as the probe approaches the centre of the defect from far away. The paths of eddy currents are disturbed by the defect which hence gives rise to a change of impedance. As the probe passes the defect centre and moves away, the second 'petal' is developed which is generally symmetrical to the first one. The shape of the trajectory is determined by the excitation frequency, lift-off distance, probe structure, the electromagnetic properties of the test specimen and the relative motion between the probe and defect. In addition, the environmental noise in a practical scenario also affects the shape. Therefore, the trajectory would have a plurality of different shapes in different actual detection situations. However, in general, the shape of trajectory would always share some similarities as an LC.

We define equations (1) and (2) for the coordinates $(x, y)$ of the complex impedance plane in which $(s, t)$ takes discrete values as expressed in (3). These equations generate $l$ number of points which form a single 'petal'. It is evident that the parameter $m$ controls the length and the parameter $\theta$ controls the inclination angle of the 'petal' which are shown in Figure 1 and 2. As shown in Figure 3, the width of the 'petal' is determined by the relative values of $a$ and $b$. For the sake of convenience, we may set $a$ as unity and the value of $b$ can be determined empirically, for instance, as a value between 0.5 and 3.5. It is noted that when $a$ equals $b$, the trajectory is a straight line segment. The other 'petal' can be generated by configuring the parameter $\theta$ as $\theta+\pi$ which is equivalent to rotating the first 'petal' by 180 degrees. Moreover, the parameters $m$ and $b$ of the first 'petal' can be assigned as a different value from that of the first 'petal' if asymmetry is desired. By alternating the values of these parameters, different LCs could be generated analytically which could provide a set of training samples. Finally, some generated LCs are illustrated in Figure 4 , from which it is demonstrated that the shape of the curve can be adjusted by manipulating the parameters.

$$
x:=m\left(\cos \theta \sin \left(s^{a}\right)-\sin \theta \sin \left(t^{b}\right)\right)
$$




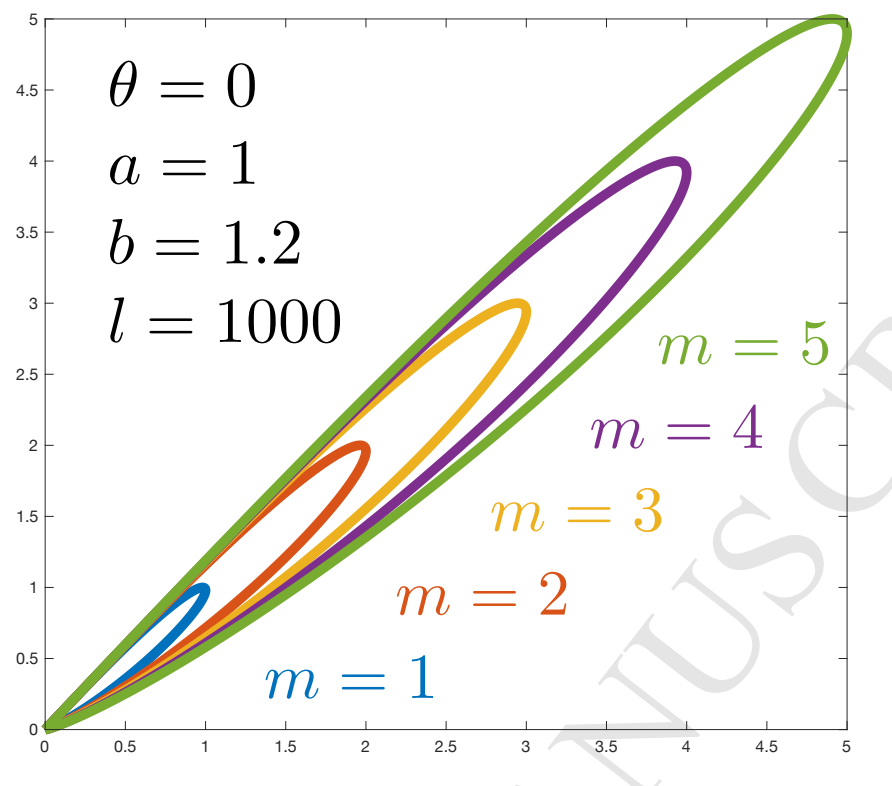

Figure 1: Plots of trajectories with different $m$.

$$
\begin{aligned}
& y:=m\left(\sin \theta \sin \left(s^{a}\right)+\cos \theta \sin \left(t^{b}\right)\right) \\
& s=\frac{\pi^{\frac{1}{a}}}{l}: \frac{\pi^{\frac{1}{a}}}{l}: \pi^{\frac{1}{a}}, t=\frac{\pi^{\frac{1}{b}}}{l}: \frac{\pi^{\frac{1}{b}}}{l}: \pi^{\frac{1}{b}}
\end{aligned}
$$

\section{Clustering-Based Feature Extraction}

In order to extract such geometric features of the LC as length, width, inclination angle and so on, we propose a clustering-based method taking advantage of the impedance data directly without using image recognition technique. The fundamental idea is that in order to extract the geometric features, we need to find some anchor points first. Then it comes naturally to exploit clustering algorithms to find some centres of the data. K-means algorithm is one of the main clustering algorithms which attempts to group the data into clusters minimising the sum of squared Euclidian distances of each data points with respect to their nearest cluster centre [13].

If we consider a typical LC as shown in Figure 5, after applying K-means clustering algorithm, three centres $C_{1}, C_{2}$ and $C_{3}$ can be found and the data are grouped into three clusters accordingly. As can be expected, the centre $C_{2}$ is very close to the crossing point of the $\mathrm{LC}$ and the other centres $C_{1}$ and $C_{2}$ extend to the two 'petals'. Therefore, the length, width and inclination angle of each 'petal' can be defined as shown in Figure 5, and they are easy to compute. Additionally, symmetric indicator features $\frac{d_{1}}{d_{2}}, \frac{w_{1}}{w_{2}}$, and $\frac{\alpha_{1}}{\alpha_{2}}$ can also be calculated. 


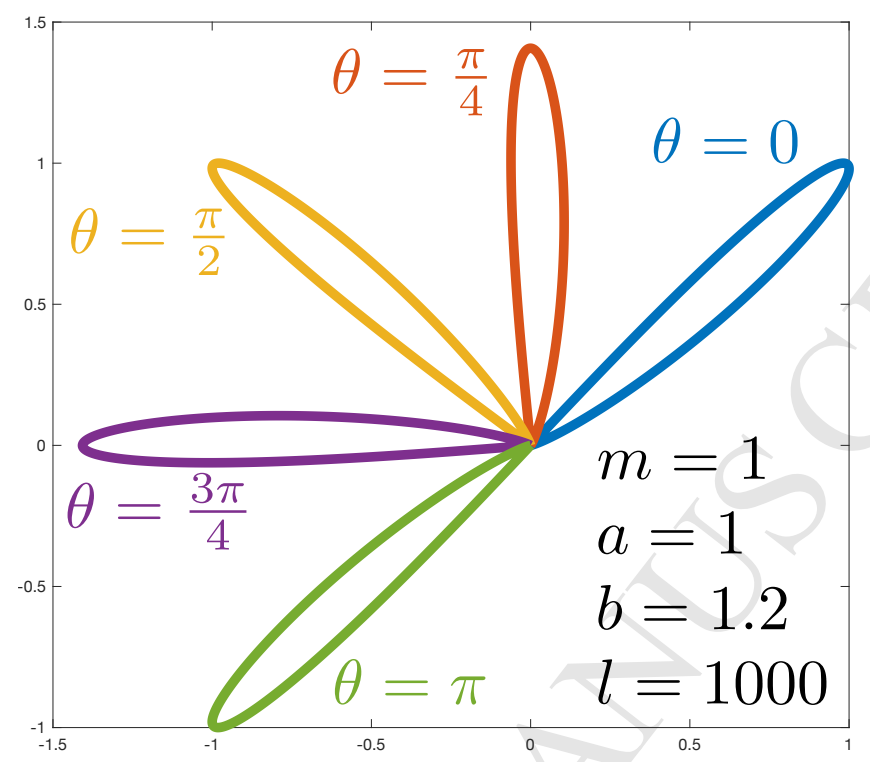

Figure 2: Plots of trajectories with different $\theta$.

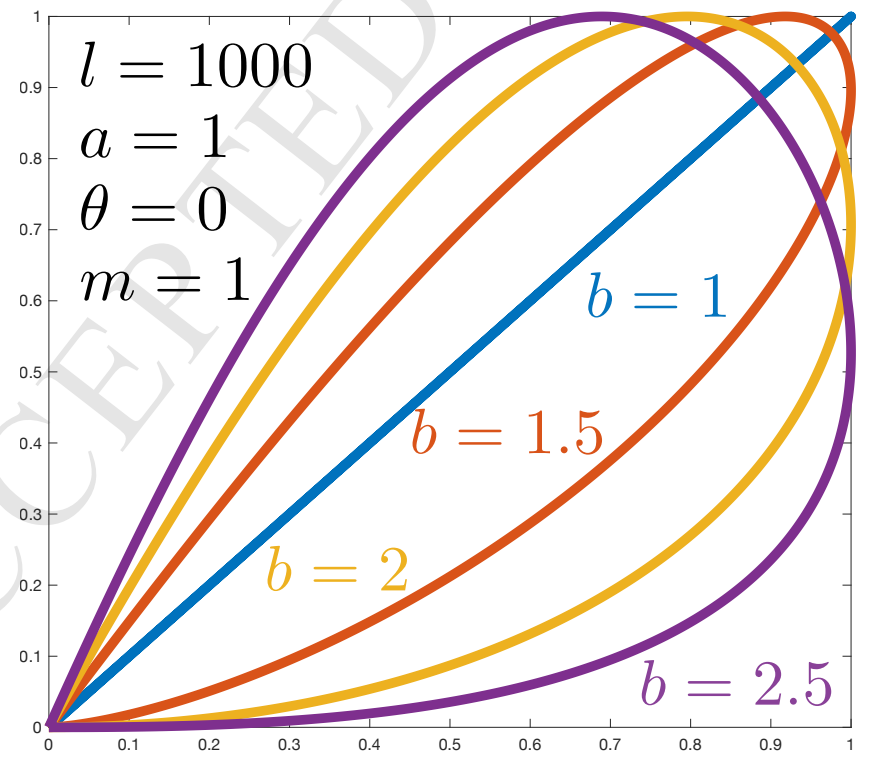

Figure 3: Plots of trajectories with different $b$. 


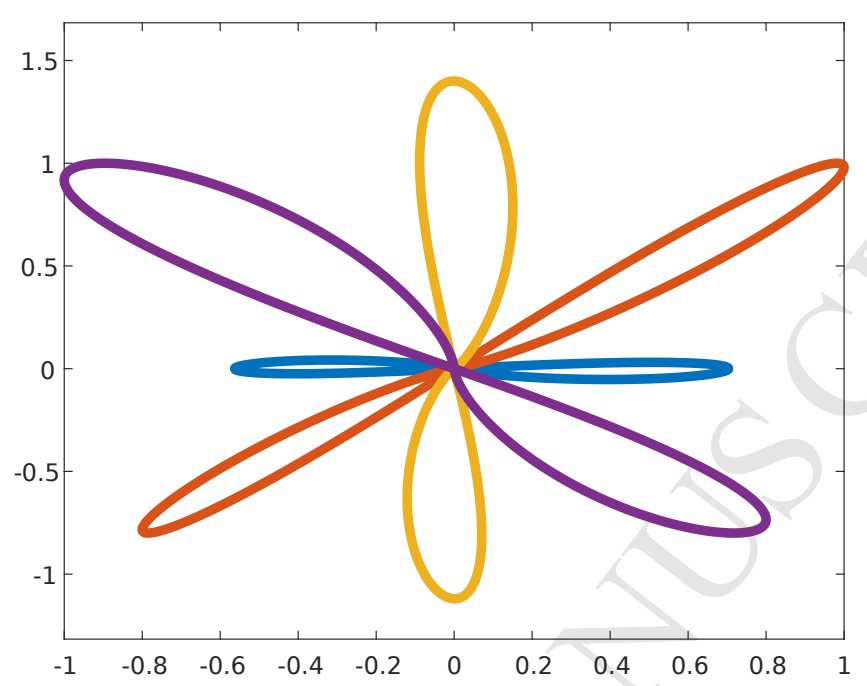

Figure 4: Plots of some generated trajectories under different $\theta, m$ and $b$.

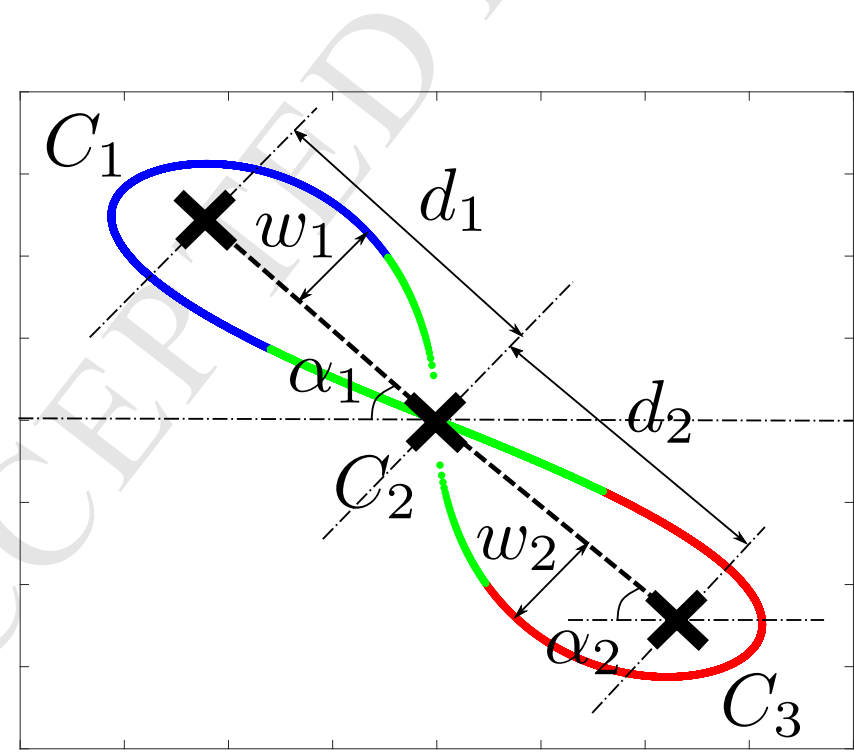

Figure 5: Clusters and geometric features of an LC. 
Now we have the tools to generate LCs and extract geometric features from them using the proposed methods.

\section{Experimental Setup}

The entire experimental system is shown in Figure 6 which comprises a scanning stage, stage controller, probe, host PC and electromagnetic (EM) instrument. The EM instrument operates in the spectrum from $5 \mathrm{kHz}$ to $200 \mathrm{kHz}$ and is capable of digital demodulation at a rate of $100 \mathrm{k}$ samples per second and has an Ethernet link to a PC. It has been successfully applied to electromagnetic sensing system such as magnetic induction tomography [33, 27] and workpiece recognition [28]. A system block diagram of the EM instrument is shown in Figure 7. The main blocks are the Zynq 7020 system on a chip (SoC), the Analog/Digital interface, the sensor front-end interface. The Zynq-7020 SoC integrates an ARM dual Cortex-A9 based processor with a Xilinx 7-series FPGA. The receiving coil is connected to the signal amplification circuit and the transmitting coil is connected to the excitation circuit. The instrument exploits the chip capabilities by implementing the signal generation and I/Q demodulation modules using the FPGA, which is typically referred as the programmable logic (PL). The ARM processor is used for data transferring between the PL and a host PC. A LabVIEW-based data acquisition and signal processing programme runs on the host PC. The scanning stage is composed of two stepper motors which drive a probe moving in two dimensions (X and $\mathrm{Y}$ ). The automatic stage is able to provide a maximum scanning speed of $100 \mathrm{~mm} / \mathrm{s}$ with a $200 \mathrm{~mm}$ travel length and $0.2 \mu \mathrm{m}$ resolution. During the experiment, the electromagnetic sensor is mounted onto a height adjustable gauge bar with a manually adjustable sensor stand by which the lift-off and direction of sensor are changeable. The resolution of the height adjustment of the gauge bar is $8 \mu \mathrm{m}$. The probe is a differential probe composed of two identical excitation coils and a receiving coil. The geometry and schematic of the probe are shown in Figure 8. The receiver coil is located between the two exciting coils. The purpose of this design is to reduce or eliminate background signals and increase the sensitivity to anomalies $[2,18]$. The system provides fast, reliable data transfer by taking advantage of the Ethernet communication. The Parameters such as amplification gain, frequency, and sampling rate provide good compatibility with varieties of sensor designs and applications. The signal-to-noise ratio (SNR) of the output signal is on average above $80 \mathrm{~dB}$. The SNR is calculated using (4) where $n$ is the number of sampling points and $z_{i}$ is the $i^{\text {th }}$ measurement.

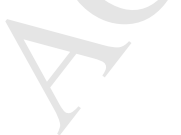

$$
S N R=10 \log _{10}\left(\frac{\sum_{i=1}^{n} z_{i}^{2}}{\sum_{i=1}^{n}\left(z_{i}-\frac{1}{n} \sum_{i=1}^{n} z_{i}\right)^{2}}\right)
$$

A steel sample $(40 \mathrm{~mm} \times 20 \mathrm{~mm} \times 2 \mathrm{~mm})$ with 20 machined slots representing simulated defects is used as the test target. The operating frequency is configured as $50 \mathrm{kHz}$. Every slot of the sample has the same length of $3 \mathrm{~mm}$ 


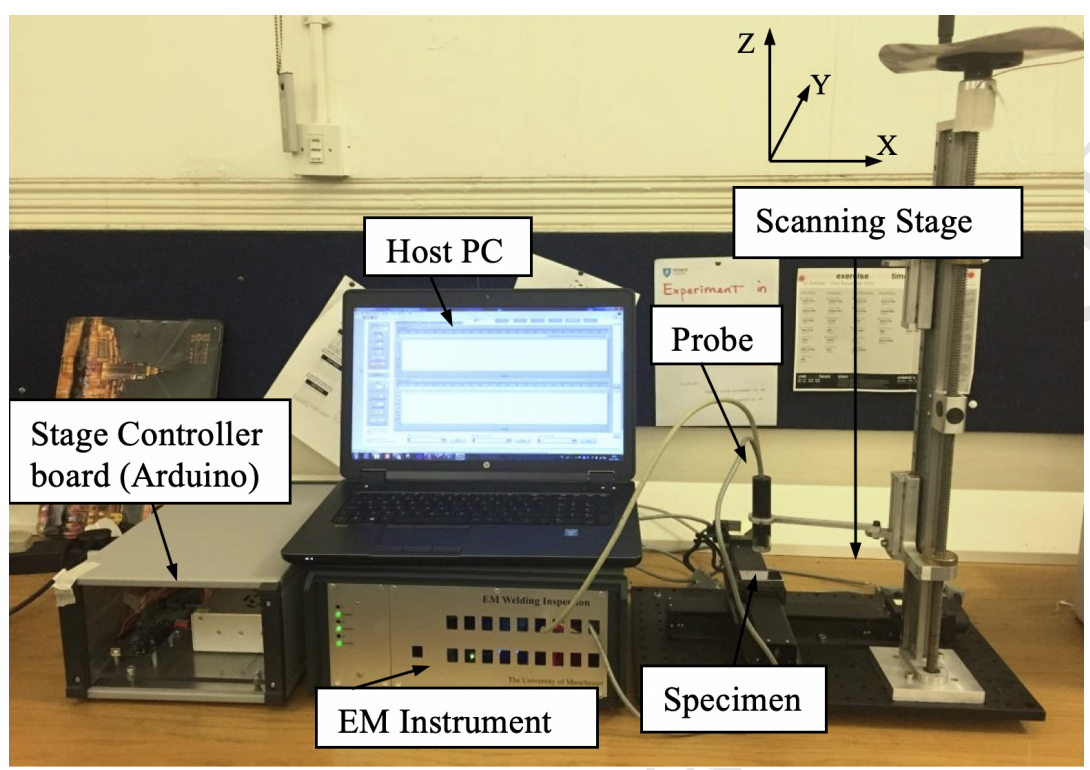

Figure 6: Experimental system.

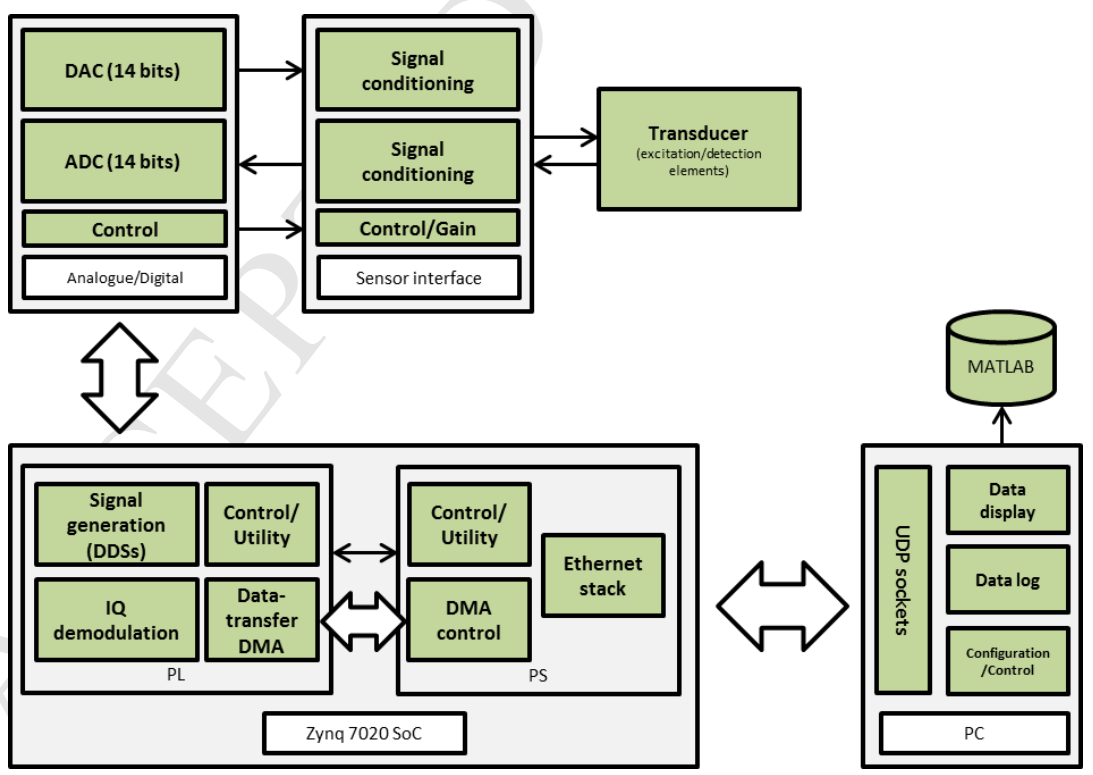

Figure 7: Block diagram of the EM instrument. 


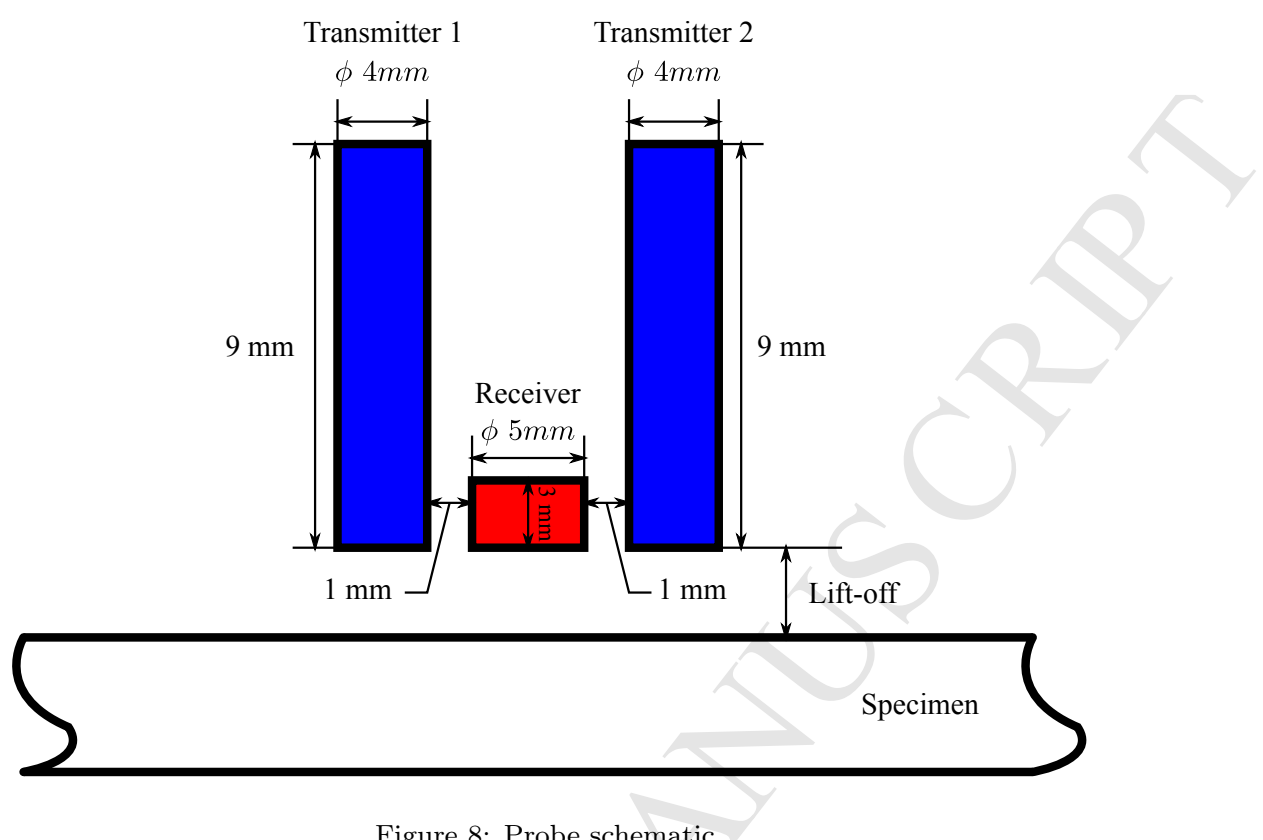

Figure 8: Probe schematic.

and width of $0.1 \mathrm{~mm}$. The depth of the slot varies from $0.1 \mathrm{~mm}$ to $2 \mathrm{~mm}$ in a step of $0.1 \mathrm{~mm}$.

Two data acquiring experiments were carried out using the experimental system in order to acquire practical scanning data of the slots and flawless area. The first experiment aimed at acquiring a standard dataset using the automatic scanning stage. In this experiment, we fixed the steel sample horizontally and the surface of open slots faced upwards. The probe was mounted onto the sensor stand and its bottom surface was parallel to the upper surface of the steel sample with a lift-off of $0.1 \mathrm{~mm}$. For every slot, the probe was programmed to scan from one flawless spot to another flawless spot crossing the slot with different angles. Such scan was carried out 50 times for every slot which resulted in 1000 LCs in total. One LC corresponding to a scan of the slot with $1.5 \mathrm{~mm}$ in depth is shown in Figure 9 (a) in which the simulated curve is plotted together with the real LC. It can be seen that in general the simulated curve aligns with the real LC. However, some discrepencies can be seen which is due to the fact the sample has a slightly different EM property even in a flat surface. Moreover, the distance of the sample and the sensor (lift-off) has a very small variation in scanning. In addition, the hardware system and sensor can be subject to EM noise in the lab during the scanning. Besides the 1000 scans of the slots, we conducted 1000 scans of the flawless area using the same setup. The flawless area was chosen randomly on the surface of the steel sample and was away from the slots and sample edges. An example of the scan of flawless area is shown in Figure 9 (b) which presents as a straight line segment. This is mainly as a result of the imperfect nilling of the differential probe signal and the unevenness of the 
steel in terms of geometry and EM properties. In total we had 2000 scans and we refer to this dataset as a standard dataset. The second experiment aimed at introducing some perturbations where the probe was held by hand and scan was carried out by hand. We tried to keep the lift-off close to $0.1 \mathrm{~mm}$ by attaching the probe to the surface of the steel sample, but perturbations existed during the experiment due to varying lift-offs and the tilt of the probe. Ten scans were carried out for every slot and 200 scans were conducted for flawless area which gave rise to a dataset containing 400 scans. Examples of the scan of the slot with $1.5 \mathrm{~mm}$ in depth and flawless area are shown in Figure 9 (c) and (d), respectively. We refer to this dataset as a perturbation dataset. Different machine learning experiments were conducted based on these two datasets.

\section{Machine Learning Process}

\subsection{Generation of artificial dataset}

Although we have the models and algorithms in Section 2 and 3 to generate an artificial dataset, there still remains such parameters as $m, \theta$ and $b$ to be determined. These parameters are obtained through calibration with an impedance plot from practical measurement. The calibration process is as follows. Firstly, an LC corresponding to a real measurement is plotted in the impedance plane, e.g. the blue points in Figure 9. Secondly, we generate points using (1), (2) and (3), and plot them on the same figure, e.g. the orange points in Figure 9. It is noted that as the differential signal is not perfectly zero, we need to add an offset, which is the mean value of the signal, to the simulated points. Thirdly, we adjust the parameters $m, \theta$ and $b$ sequentially to align the simulated LC with the real LC. Lastly, the parameters $m, \theta$ and $b$ are perturbed by adding a random value within $\pm 10 \%$ of their values obtained from the third step in order to generate an artificial LC. Applying this calibration process to every LC in the standard and perturbation dataset, artificial datasets are obtained.

\subsection{ML algorithms and Bayesian optimisation on hyper-parameters}

In this paper, six prevailing supervised-learning classifiers are adopted and they are the Classification Trees (CT) [16], Discriminant Analysis (DA) [14], Naive Bayes (NB) [15], K-Nearest Neighbours (KNN) [31], Support Vector Machine (SVM) [19], and Classification Ensemble (CE) [21]. These algorithms are representatives of different classification strategies. In terms of the CT classifier, decisions are made following a tree structure from a root node to leaf nodes. Training a CT classifier is equivalent to growing a tree minimising the sum of classification errors over each training sample. While the DA algorithm takes a different point of view that the feature data of different classes are generated from different Gaussian distributions. Therefore, to train a DA classifier, the fitting function estimates the parameters of a multivariate Gaussian distribution for each class. In contrast, the NB algorithm assumes that the features are conditionally independent and therefore individual density distributions are calculated for each feature based on training samples. With respect to the KNN 


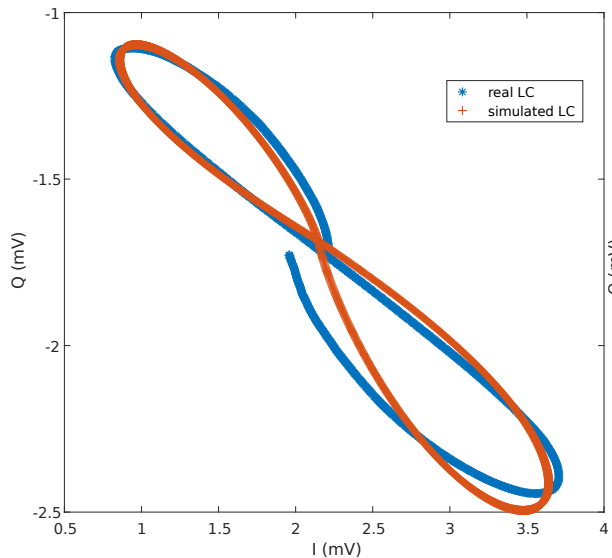

(a)

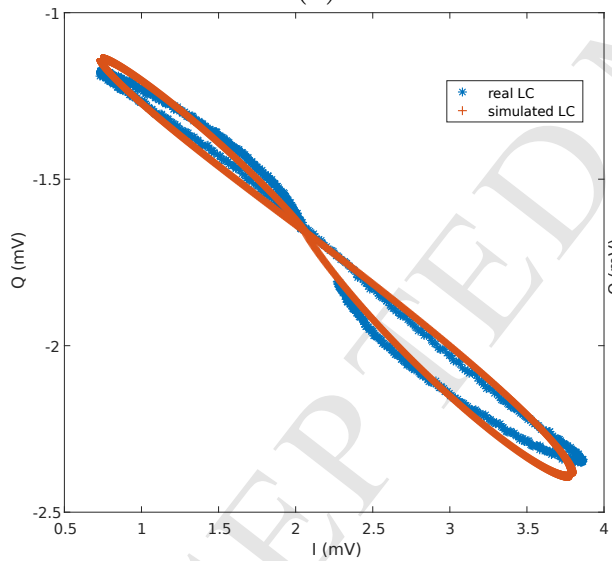

(c)

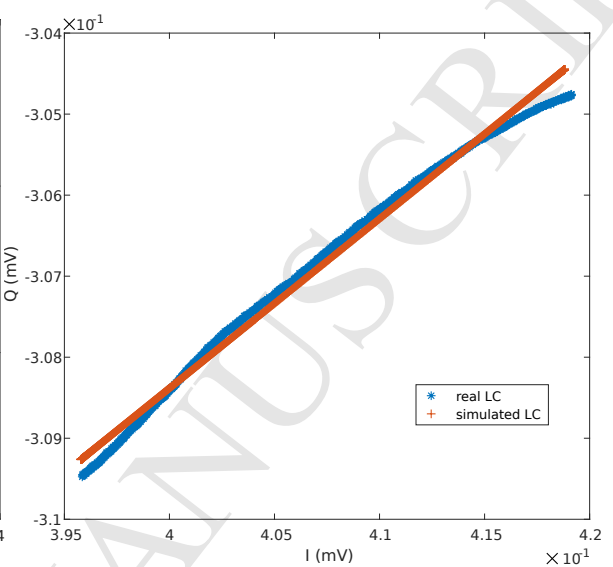

(b)

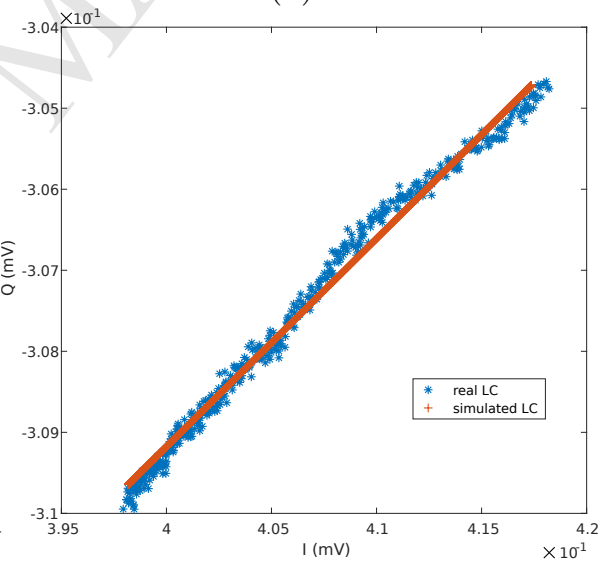

(d)

Figure 9: Trajectory of real and simulated data in the complex plane: (a) scan of the slot with $1.5 \mathrm{~mm}$ in depth in the standard dataset; (b) scan of flawless area in the standard dataset; (c) scan of the slot with $1.5 \mathrm{~mm}$ in depth in the perturbation dataset; (d) scan of flawless area in the perturbation dataset . 
model, distance is adopted as a metric to find $\mathrm{K}$ neighbours for a sample point and classification is made based upon the labels of the neighbours. In terms of the SVM algorithm, a hyper-plane is pursuit that maximises the margin separating the two class points. The $\mathrm{CE}$ is constructed by a combination of the previous multiple classification models with different weights. In addition to the ML classifiers, choosing proper hyper-parameters for these models is an important factor that affects the performance of the classifiers. Bayesian optimisation [24] is carried out for the training of each classifier which minimises the five-fold cross-validation error of the training set.

\subsection{Error analysis}

Based on the confusion matrix, different error quantities are available in the evaluation of the performance of ML models. In this paper, we adopt the classification error [26], which is defined as the number of incorrect classifications divided by the total number of samples, as the metric to choose hyper-parameters and evaluate the accuracy of training and prediction.

\section{Result and Discussion}

Using the LC generating methods proposed in Section 2 and applying the data augmentation process in Section 5.1, ten simulated LCs were generated for every LC in the standard dataset which gave rise to an artificial standard set containing 20000 samples. These simulated and empirical data were fed into the K-means clustering algorithm discussed in Section 3, after which geometric features were extracted. Then two experiments were conducted in order to validate the proposed analytic model and feature extraction method.

The first experiment harnessed the real and artificial standard set. The fundamental idea was that one group of ML models presented in Section 5.2 were trained using the real dataset, and another group of ML models were trained using the artificial dataset, and then we evaluated the two groups of trained ML models using the data in the real dataset that were not used in the training of ML models. By comparing the classification accuracies of these two groups of ML models with respect to the real test data, a conclusion could be drawn that whether the artificial dataset was able to represent the real dataset. Detailed division of the standard datasets were as follow. In terms of the real dataset, we randomly selected 40 scans of every slot as positive samples of the real training set and hence there were 800 positive samples. In addition, 800 scans of flawless area in the real dataset were randomly chosen as negative samples. In total, we had 1600 samples in the real training set. The remaining 400 samples in the real dataset were utilised as a test set. With respect to the artificial training set, we selected 16000 samples corresponding to the 1600 samples of the real training set. It is noted that the remaining 4000 artificial samples corresponding to the 400 real test samples were not used in this experiment.

The classification accuracies of the six trained ML models in terms of the real and artificial training set are listed in Table 1 and 2, respectively. From 


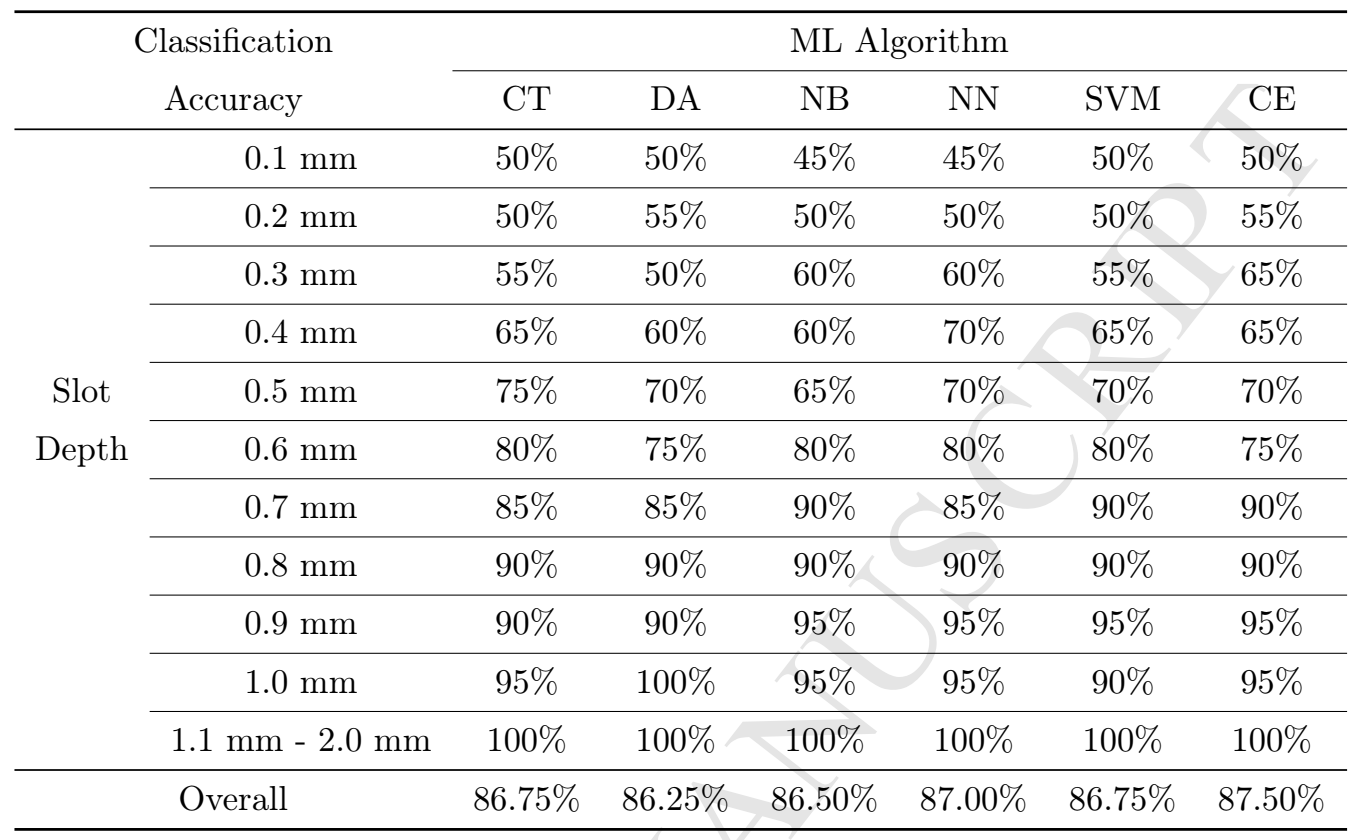

Table 1: Classification accuracies of the ML algorithms in terms of the real training set.

Table 1 it can be seen that as the depth of the slot increases, the classification accuracy becomes higher. This trend applies to all the six ML algorithms in general. When the depth of slot is small, e.g. $0.2 \mathrm{~mm}$, the detection rate is low and concretely many test samples are predicted as flawless. In contrast, $100 \%$ classification accuracies are achieved for the slots of depth from $1.1 \mathrm{~mm}$ to $2.0 \mathrm{~mm}$. The six ML models have achieved similar classification accuracies in terms of the overall performance. If we compare Table 1 and 2, we can find that the classifications accuracies are very similar. This result implies that on the one hand, the artificial dataset highly approximates the real dataset as the ML models trained based on them give close predictions of the test samples, which proves the validity of the proposed analytical model and feature extraction method. One the other hand, the artificial dataset does not improve the classification accuracy although it has more data than the real dataset. This can be as a result of the fact that the calibration process for the generation of the artificial dataset relies on the real dataset and the perturbation of the parameters of the analytical model fails to introduce new variance of the feature space that targets the test set.

In the second experiment, we would like to evaluate the trained ML models obtained in the first experiment with the perturbation dataset as explained in Section 4. By conducting this experiment, we can examine the robustness of the models to perturbations such as inconsistent lift-off and probe tilt. The classification accuracies of the test samples of perturbation set in terms of the ML models trained using the real standard set and the artificial standard set 


\begin{tabular}{|c|c|c|c|c|c|c|c|}
\hline & Classification & & & ML Algo & orithm & & \\
\hline & Accuracy & $\mathrm{CT}$ & DA & NB & NN & SVM & $\mathrm{CE}$ \\
\hline & $0.1 \mathrm{~mm}$ & $50 \%$ & $50 \%$ & $50 \%$ & $50 \%$ & $50 \%$ & $50 \%$ \\
\hline & $0.2 \mathrm{~mm}$ & $50 \%$ & $55 \%$ & $50 \%$ & $50 \%$ & $50 \%$ & $55 \%$ \\
\hline & $0.3 \mathrm{~mm}$ & $60 \%$ & $55 \%$ & $55 \%$ & $55 \%$ & $60 \%$ & $65 \%$ \\
\hline & $0.4 \mathrm{~mm}$ & $70 \%$ & $65 \%$ & $65 \%$ & $65 \%$ & $60 \%$ & $65 \%$ \\
\hline Slot & $0.5 \mathrm{~mm}$ & $70 \%$ & $75 \%$ & $70 \%$ & $70 \%$ & $65 \%$ & $70 \%$ \\
\hline Depth & $0.6 \mathrm{~mm}$ & $80 \%$ & $80 \%$ & $80 \%$ & $80 \%$ & $80 \%$ & $75 \%$ \\
\hline & $0.7 \mathrm{~mm}$ & $90 \%$ & $90 \%$ & $90 \%$ & $85 \%$ & $90 \%$ & $90 \%$ \\
\hline & $0.8 \mathrm{~mm}$ & $90 \%$ & $90 \%$ & $90 \%$ & $90 \%$ & $95 \%$ & $90 \%$ \\
\hline & $0.9 \mathrm{~mm}$ & $95 \%$ & $90 \%$ & $100 \%$ & $90 \%$ & $95 \%$ & $95 \%$ \\
\hline & $1.0 \mathrm{~mm}$ & $95 \%$ & $95 \%$ & $95 \%$ & $100 \%$ & $95 \%$ & $95 \%$ \\
\hline & $1.1 \mathrm{~mm}-2.0 \mathrm{~mm}$ & $100 \%$ & $100 \%$ & $100 \%$ & $100 \%$ & $100 \%$ & $100 \%$ \\
\hline & Overall & $87.50 \%$ & $87.25 \%$ & $87.25 \%$ & $86.75 \%$ & $87.00 \%$ & $87.75 \%$ \\
\hline
\end{tabular}

Table 2: Classification accuracies of the ML algorithms in terms of the artificial training set.

\begin{tabular}{ccccccc}
\hline \multirow{2}{*}{ Classification Accuracy } & \multicolumn{7}{c}{ ML Algorithm } \\
\cline { 2 - 7 } & CT & DA & NB & NN & SVM & CE \\
\hline Real Training Set & $70.50 \%$ & $68.50 \%$ & $69.00 \%$ & $67.00 \%$ & $71.25 \%$ & $71.50 \%$ \\
\hline Artificial Training Set & $75.50 \%$ & $73.25 \%$ & $74.50 \%$ & $76.00 \%$ & $77.50 \%$ & $76.50 \%$ \\
\hline
\end{tabular}

Table 3: Classification accuracies of the ML algorithms testing the perturbation dataset.

are listed in Table 3. From the table, it is observed that lower detection rates are obtained for both groups of ML models compared to the detection rates of the standard test set. However, in general, the ML models trained using the artificial standard set have higher accuracies in predicting the labels of the samples of the perturbation set. This phenomenon indicates that augmenting the training set by harnessing the analytical model and perturbing the model parameters can be useful in introducing some robustness to the dataset.

\section{Conclusion}

Recent ECT studies tend to take advantage of the fast developing machine learning techniques. Data augmentation is an interesting topic when there are limited resources to acquire data. This paper has proposed an analytical method to generate Lissajous curves which would give rise to an artificial 
dataset. Moreover, since feature extraction is an important procedure for training ML models, a clustering-based method has been proposed to extract the geometric feature of LCs. Further, six machine learning-based classifiers are used for automatic defect identification based on these generated training data. High detection rates are achieved for both the simulated and experimental data, which demonstrates the flexibility of the analytical model and the validity of the methodology.

\section{Acknowledgement}

This work is supported by the National Natural Science Foundation of China (Grant No. 51465024) and the UK Engineering and Physical Sciences Research Council (EPSRC, Grant No. EP/M020835/1).

\section{Reference}

[1] A. Bernieri, L. Ferrigno, M. Laracca, and M. Molinara. Crack Shape Reconstruction in Eddy Current Testing Using Machine Learning Systems for Regression. IEEE Transactions on Instrumentation and Measurement, 57 (9):1958-1968, September 2008.

[2] G. Betta, L. Ferrigno, M. Laracca, H. G. Ramos, M. Ricci, A. L. Ribeiro, and G. Silipigni. Fast $2 \mathrm{~d}$ crack profile reconstruction by image processing for Eddy-Current Testing. In 2015 IEEE Metrology for Aerospace (MetroAeroSpace), pages 341-345, June 2015.

[3] M. Bodruzzaman and S. Zein-Sabatto. Estimation of micro-crack lengths using eddy current C-scan images and neural-wavelet transform. In IEEE SoutheastCon 2008, pages 551-556, April 2008.

[4] J. A. Buck, P. R. Underhill, J. E. Morelli, and T. W. Krause. Simultaneous Multiparameter Measurement in Pulsed Eddy Current Steam Generator Data Using Artificial Neural Networks. IEEE Transactions on Instrumentation and Measurement, 65(3):672-679, March 2016.

[5] T. Chady and P. Lopato. Flaws Identification Using an Approximation Function and Artificial Neural Networks. IEEE Transactions on Magnetics, 43(4):1769-1772, April 2007.

[6] Tianlu Chen, Gui Yun Tian, Ali Sophian, and Pei Wen Que. Feature extraction and selection for defect classification of pulsed eddy current NDT. NDT \& E International, 41(6):467-476, September 2008.

[7] G. D'Angelo and S. Rampone. Shape-based defect classification for non destructive testing. In 2015 IEEE Metrology for Aerospace (MetroAeroSpace), pages 406-410, June 2015. 
[8] G. D'Angelo, M. Laracca, and S. Rampone. Automated Eddy Current nondestructive testing through low definition lissajous figures. In 2016 IEEE Metrology for Aerospace (MetroAeroSpace), pages 280-285, June 2016.

[9] G. D'Angelo, M. Laracca, S. Rampone, and G. Betta. Fast Eddy Current Testing Defect Classification Using Lissajous Figures. IEEE Transactions on Instrumentation and Measurement, 67(4):821-830, April 2018.

[10] Yunze He, Mengchun Pan, Feilu Luo, and Guiyun Tian. Pulsed eddy current imaging and frequency spectrum analysis for hidden defect nondestructive testing and evaluation. NDT \& E International, 44(4):344352 , July 2011.

[11] Yunze He, Mengchun Pan, Dixiang Chen, and Feilu Luo. PEC defect automated classification in aircraft multi-ply structures with interlayer gaps and lift-offs. NDT $\&$ E International, 53:39-46, January 2013.

[12] Saleh Hosseini and Aouni A. Lakis. Application of time-frequency analysis for automatic hidden corrosion detection in a multilayer aluminum structure using pulsed eddy current. NDT \& E International, 47:70-79, April 2012 .

[13] T. Kanungo, D. M. Mount, N. S. Netanyahu, C. D. Piatko, R. Silverman, and A. Y. Wu. An Efficient k-Means Clustering Algorithm: Analysis and Implementation. IEEE Transactions on Pattern Analysis 83 Machine Intelligence, 24(7):881-892, 2002.

[14] P. A. Lachenbruch and M. Goldstein. Discriminant Analysis. Biometrics, 35(1):69-85, 1979.

[15] David D. Lewis. Naive (Bayes) at forty: The independence assumption in information retrieval. In Claire Nédellec and Céline Rouveirol, editors, Machine Learning: ECML-98, Lecture Notes in Computer Science, pages 4-15. Springer Berlin Heidelberg, 1998.

[16] Wei-Yin Loh. Classification and regression trees. Wiley Interdisciplinary Reviews: Data Mining and Knowledge Discovery, 1(1):14-23, January 2011.

[17] L.A.N.M. Lopez, D.K.S. Ting, and B.R. Upadhyaya. Removing EddyCurrent probe wobble noise from steam generator tubes testing using Wavelet Transform. Progress in Nuclear Energy, 50(7):828-835, September 2008.

[18] Y. Nagaya, T. Takagi, T. Uchimoto, and Haoyu Huang. Identification of multiple cracks from eddy-current testing signals with noise sources by image processing and inverse analysis. IEEE Transactions on Magnetics, 40(2):1112-1115, March 2004.

[19] Edgar Osuna, Robert Freund, and Federico Girosi. Support Vector Machines: Training and Applications. March 1997. 
[20] Rainer Pohl, A Erhard, H. J Montag, H. M Thomas, and H Wüstenberg. NDT techniques for railroad wheel and gauge corner inspection. NDT $\mathscr{E} E$ International, 37(2):89-94, March 2004.

[21] Lior Rokach. Ensemble-based classifiers. Artificial Intelligence Review, 33 (1):1-39, February 2010.

[22] L. S. Rosado, F. M. Janeiro, P. M. Ramos, and M. Piedade. Defect Characterization With Eddy Current Testing Using Nonlinear-Regression Feature Extraction and Artificial Neural Networks. IEEE Transactions on Instrumentation and Measurement, 62(5):1207-1214, May 2013.

[23] Sikora, Ryszard, Mieczyslaw Komorowski, and Tomasz Chady. A neural network model of eddy current probe. Stuides in Applied Electromagntics and Mechanics, pages 213-238, 1997.

[24] Jasper Snoek, Hugo Larochelle, and Ryan P Adams. Practical Bayesian Optimization of Machine Learning Algorithms. In F. Pereira, C. J. C. Burges, L. Bottou, and K. Q. Weinberger, editors, Advances in Neural Information Processing Systems 25, pages 2951-2959. Curran Associates, Inc., 2012.

[25] G. Sposito, C. Ward, P. Cawley, P. B. Nagy, and C. Scruby. A review of non-destructive techniques for the detection of creep damage in power plant steels. NDT $\&$ E International, 43(7):555-567, October 2010.

[26] Patrick Stalph. Analysis and Design of Machine Learning Techniques. Springer Fachmedien Wiesbaden, 2014.

[27] Y. Tao, W. Zhang, Y. Zhao, C. Ktistis, W. Yin, and A. J. Peyton. Sparse electromagnetic tomography based on matching pursuit algorithms. In 2016 IEEE International Instrumentation and Measurement Technology Conference Proceedings, pages 1-6, May 2016.

[28] Y. Tao, W. Yin, W. Zhang, Y. Zhao, C. Ktistis, and A. J. Peyton. A Very-Low-Frequency Electromagnetic Inductive Sensor System for Workpiece Recognition Using the Magnetic Polarizability Tensor. IEEE Sensors Journal, 17(9):2703-2712, May 2017.

[29] G. Y. Tian, A. Sophian, D. Taylor, and J. Rudlin. Wavelet-based PCA defect classification and quantification for pulsed eddy current NDT. IEE Proceedings - Science, Measurement and Technology, 152(4):141-148, July 2005.

[30] Gui Yun Tian and Ali Sophian. Defect classification using a new feature for pulsed eddy current sensors. NDT \& E International, 38(1):77-82, January 2005. 
[31] Enrique Vidal Ruiz. An algorithm for finding nearest neighbours in (approximately) constant average time. Pattern Recognition Letters, 4(3):145157, July 1986.

[32] Maria Wrzuszczak and Janusz Wrzuszczak. Eddy current flaw detection with neural network applications. Measurement, 38(2):132-136, September 2005 .

[33] Wuliang Yin, Guang Chen, Lijing Chen, and Ben Wang. The Design of a Digital Magnetic Induction Tomography (MIT) System for Metallic Object Imaging Based on Half Cycle Demodulation. IEEE Sensors Journal, 11 (10):2233-2240, October 2011. 